\title{
Transesophageal echocardiography detects an unusual complication of infective endocarditis
}

\author{
Sophia S. F. Wong, MD
}

Received: 21 April 2009/ Accepted: 10 September 2009/Published online: 7 October 2009

(C) Canadian Anesthesiologists' Society 2009

With infective endocarditis, infection can spread to the perivalvular structures. Occasionally, aortic abscesses can rupture into adjacent cardiac chambers and lead to aortocavitary fistulas. ${ }^{1,2}$ We highlight images involving a case where intraoperative transesophageal echocardiography played a pivotal role in the surgical management of a male patient who presented with a three-month history of progressive dyspnea. He had a history of disseminated tuberculosis, deep vein thrombosis, and chronic renal insufficiency secondary to glomerulonephritis. Transthoracic echocardiography revealed severe aortic regurgitation with a jet crossing a fenestration in an aneursymal sinus of valsalva, as well as moderate global hypokinesis of the left ventricle. The patient was scheduled for aortic valve replacement. However, an intraoperative transesophageal echocardiogram revealed a normally appearing trileaflet aortic valve. The sinuses of valsalva did not appear aneurysmal. A false aneurysm of the aortic annulus was protruding into the left ventricular outflow tract with severe aortic regurgitation (Figs. 1, 2) (video images available as Supplemental Material at www.springerlink.com/12630), suggesting that replacement of the aortic valve was not necessarily indicated.

Surgical findings showed a defect at the base of the annulus at the level of the origin of the right coronary leaflet, not involving the right coronary artery (video images available as Supplemental Material at www.springerlink.com/ 12630). Through the defect, the surgeon observed the

Electronic supplementary material The online version of this article (doi:10.1007/s12630-009-9193-8) contains supplementary material, which is available to authorized users.

S. S. F. Wong, MD ( $\square)$

Sunnybrook Health Sciences Centre, Toronto, Canada

e-mail: sophia.wong@sunnybrook.ca

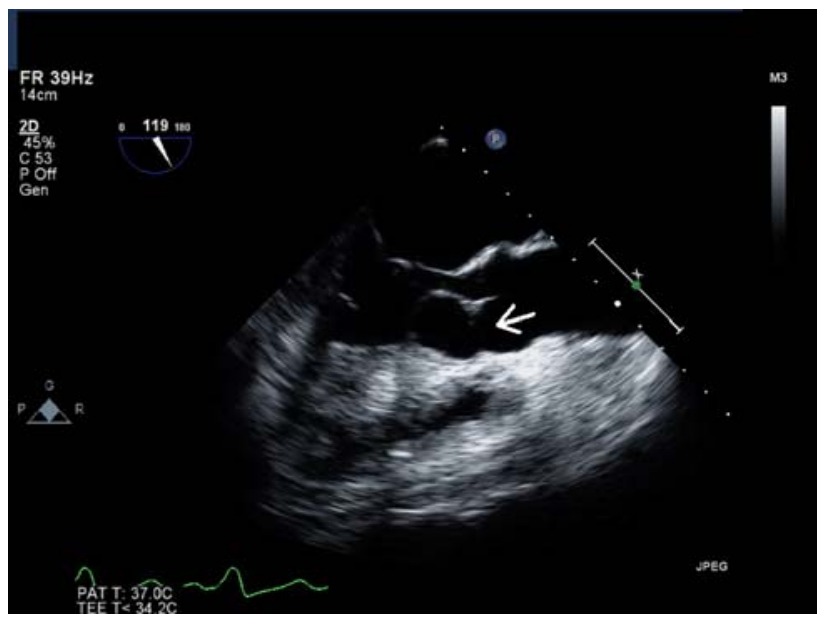

Fig. 1 Mid-esophageal aortic valve long-axis view showing the defect as indicated by the arrow

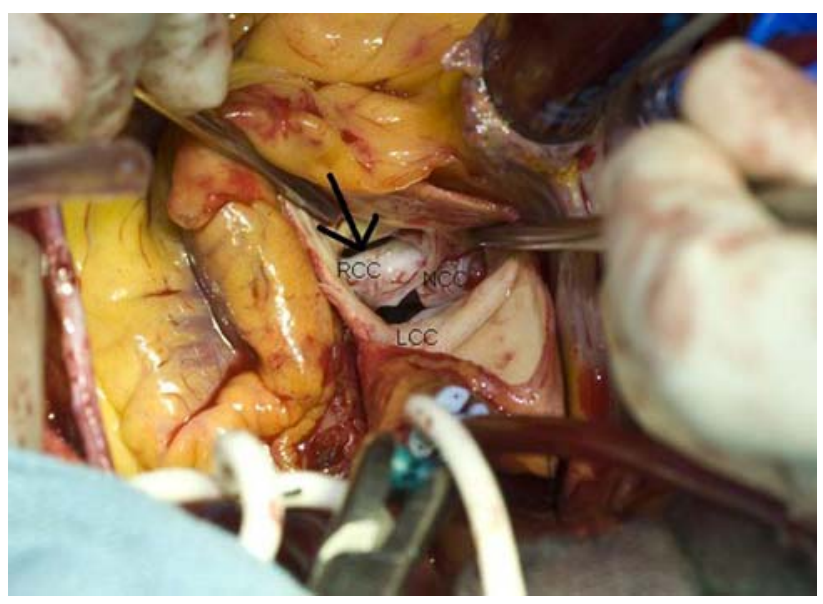

Fig. 2 Photograph showing the defect of the aortic valve annulus. $R C C$ right coronary cusp, $N C C$ non-coronary cusp, $L C C$ left coronary cusp. Arrow highlights the defect 
existence of a false aneurysm passing through approximately $7.5 \mathrm{~cm}$ into the left ventricle. The valve leaflets were otherwise normal. Despite negative blood cultures, this finding was considered to be consistent with previous endocarditis. Valvuloplasty with resuspension of the valve and repair of the defect with bovine pericardial patch was performed. Transesophageal echocardiography performed post-cardiopulmonary bypass showed minimal central aortic regurgitation. The patient recuperated well and he was discharged on postoperative day six.
Competing interests None declared.

\section{References}

1. Anguera I, Quaglio G, Miro JM, et al.. Aortocardiac fistulas complicating infective endocarditis. Am J Cardiol 2001; 87: 652-4.

2. Anguera I, Miro JM, Evangelista A, et al. Periannular complications in infective endocarditis involving native aortic valves. Am J Cardiol 2006; 98: 1254-60. 